\title{
Hard materials used in mechanical heart valves: A critical review
}

\author{
Abhimanyu Tharayil, ${ }^{1}$ Vivek Verma, ${ }^{1}$ Malay K. Das, ${ }^{2}$ and Kamal K. Kar ${ }^{2,3^{*}}$ \\ ${ }^{1}$ Department of Materials Science and Engineering, Indian Institute of Technology Kanpur, Kanpur- \\ 208016, UP, India \\ ${ }^{2}$ Department of Mechanical Engineering, Indian Institute of Technology Kanpur, Kanpur-208016, \\ India \\ ${ }^{3}$ Advanced Nanoengineering Materials laboratory, Materials Science Programme, Indian Institute of \\ Technology Kanpur, Kanpur-208016, India \\ *Email: kamalkk@iitk.ac.in
}

The first mechanical heart valve replacement surgery was done in 1960 by Dr. Albert Starr and Lowell Edwards. It is estimated that annually around 300,000 artificial heart valves are being substituted and around $45 \%$ of them are mechanical valves and the rest are bioprosthetic valves. The former have longterm durability but lifelong anticoagulation is required with high risk of thrombosis. Biological valves are usually constructed from pig or cow tissues and are specially treated so that the body does not reject them. However, they have a smaller lifetime compared to their mechanical counterparts [1]. Even now after around 50 years after the first mechanical heart valve replacement there was never a quantum leap in the advancement of these prosthesis. Mechanical valves still possess the risk of life long anti-coagulation therapy and tissue valves are not durable and are mainly implanted in older patients. The procedure of re-operating the patient repeatedly for replacing the heart valve in itself is not a healthy process.

The choice of material plays a very important role, as in it should be able to withstand the harsh and corrosive environment of the body and should not lead to rejection. Albert Starr implanted a heart valve in 1961, which had a metallic cage and silicon ball. Stellite- 21 was the material choice for the cage and is an alloy of cobalt, chromium, molybdenum and nickel and showed good biocompatibility. The LK tilting disc introduced in 1970 had an orifice ring that was machined by $\mathrm{CNC}$, from highgrade titanium, and was hand polished to obtain a mirror finish. The occluders were made of pyrolytic carbon (PyC), which had a flat disk.
Later on, there were major structural improvements in which the curved occluder disk was introduced and the orifice block was made from pyrolytic carbon. The St Jude Bileaflet valve introduced in 1976 had two semi-circular leaflets.

Here the forward flow region is divided into three regions; it had two lateral orifices wherein majority of the flow takes place and a central orifice [2, 3]. Latest development is the On-X valve, which is of bi-leaflet design, and is made up of graphite and tungsten and are carbon coated. Figure 1 . shows the mechanical valves through years and their materials (poppet, housing)

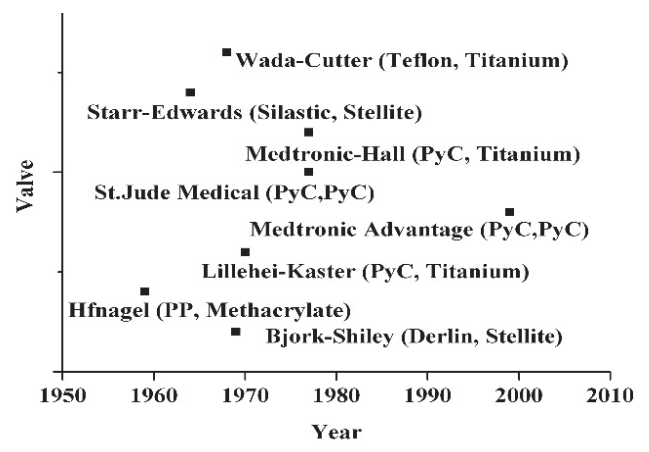

Figure 1: Mechanical valves through years with material used (poppet material, housing material)

\section{References}

1. Yacoub MH, Cohn LH. Circulation 2004; 109:942-950

2. Hufnagel CA. Reflections on the development of valvular prostheses. Med Instrum 1977;11: 746

3. Richard A. DeWall, Naureen Qasim, and Liz Carr, Ann Thorac Surg 2000;69: 1612-21 